\title{
A century and a half of plant physiology in Saint Petersburg
}

In December 2017 we celebrated the $150^{\text {th }}$ anniversary of the Department of Plant Physiology and Biochemistry of St. Petersburg State University. This special issue of Biological Communications is dedicated to the overview of the rich history of one of the oldest departments of plant physiology in the world and the representation of the current state of research in the fascinating field of plant biology. Avalanche-like advances in state-of-the-art biological technologies evoke growing interest of researchers in the fundamental problems of plant life and the prospect of application of these technologies in agriculture, conservation and ecological monitoring. Several reviews and experimental articles presented in this issue describe various aspects of research in plant biology, including plant response to stresses, growth and development, intracellular organization of plant cells, plant metabolites and proteins.

The issue begins with an overview of the history of the department by its current head professor Sergei Medvedev, and by Gregory Pozhvanov. In the late $19^{\text {th }}-$ early $20^{\text {th }}$ centuries, famous Russian plant biologists Andrej Famintsyn, Dimitri Neljubow, Vladimir Palladin, and Sergei Kostychev created a foundation for the first plant physiology and biochemistry department in Russia. Their classical discoveries inspired many scientific schools throughout the country whose activities greatly contributed to current progress in plant physiology and biochemistry.

Currently, physiology and biochemistry of plants is among the most intensively developing areas in biology. Current agricultural and environmental challenges set new global questions for humanity. To solve them, plant biology employs the principles of systems biology based on modern state-of-the-art technologies, including 'omics' approaches, which are widely used at the department. Advances in modern omics technologies have prompted progress in studies of the transciptome, proteome and metabolome. A perfect example is a paper by Andrej Frolov and co-authors devoted to seed proteomics as one of the most effective tools to study physiological changes related to environmental stress and ageing. This review is a result of fruitful collaboration of departments of St. Petersburg State University, the All-Russia Research Institute for Agricultural Microbiology and several institutions in Germany, initiated by Professor Sergei Medvedev.

Target transcriptomics was employed in the study by Roman Puzanskiy and co-authors. They evaluated the importance of the genes encoding enzymes of central metabolism and plastid transporters for the exponential growth of Chlamydomonas reinhardtii autotrophic culture.

Algal physiology and biochemistry has been a traditional area of research at the department since the 1960s. This research is carried out at the department of St. Petersburg University and its affiliate at the White Sea Marine Biological Station. The review by Valeriya Lemesheva and Elena Tarakhovskaya describes phlorotannins, plant metabolites synthesized by brown algae. These compounds, which could be detected by target metabolomics, have a specific chemical structure and possess high biological activities.

Besides omics technologies, another scientific approach in high demand is visualisation of different molecules, cellular structures and cellular events with the use of confocal fluorescent microscopy. Different techniques for actin visualization are reviewed by Gregory Pozhvanov. Actin microfilaments are essential for polar growth, cytoplasmic streaming, gravity sensing, signalling pathway integration and a number of other functions.

Citation: Minibayeva, F. and Shishova, M. 2018. A century and a half of plant physiology in Saint Petersburg (Editorial). Bio. Comm. 63(1): 3-4. https://doi. org/10.21638/spbu03.2018.101 
Diverse modern techniques of plant cellular biology are intensively used in Professor Maria Shishova's group to study the mechanisms of signalling of the phytohormone auxin. This research was initiated by Professor Vsevolod Polevoi. The paper by Anastasia Kirpichnikova and co-authors describes the historical background and modern solution to this problem. In the review, a possible scheme of the regulation of elongation growth, based on the shift in plant cell sensitivity to auxin and the activity of the plasma membrane proton pump, is suggested.

Elongation growth is controlled by processes that occur not only inside the cell, but also in the cell wall. Elena Sharova and Aleksandra Romanova describe recent experimental data on apoplastic ascorbate, per- oxidase activity and redox state in the growth zone of etiolated maize mesocotyls.

Another traditional area of research at the department is conducted by the group led by Professor Tamara Chirkova. Beginning in the early 1960s, she and her colleagues established a new scientific direction at the department, devoted to the study of plant adaptation to oxygen deprivation. The milestones of this work are reviewed by Tamara Chirkova and Vladislav Yemelyanov.

Abiotic stresses induce numerous morphological, physiological, biochemical and molecular changes in plants, which affect their growth and productivity. Effects of heavy metal stress on plants are described in the paper by Natalia Osmolovskaya and co-authors. The authors put special emphasis on the importance of organic acids in providing heavy metal tolerance to plants. The study of organic acid metabolism and mineral nutrition of plants is another traditional research area in the department; this research was begun in the 1930s by Professors Stepan Soldatenkov and Vladimir Chesnokov.

Taken together, the reviews and experimental papers presented in this special issue demonstrate the continuous progress of the department now celebrating its $150^{\text {th }}$ anniversary. An abundance of approaches, wide collaboration, financial support and attractiveness for young scientists and students give strong confidence in the future of plant functional biology at St. Petersburg State University.

Farida V. Minibayeva, Section Editor for Plant Physiology

Maria F. Shishova, Guest Editor of "Biological Communications" 